\title{
MEIOSIS IN SPIROGYRA CRASSA
}

\author{
M. B. E. GODWARD \\ Department of Botany, Queen Mary College, London
}

Received 12.x.6o

\section{THE PROBLEM}

AMoNG those organisms known not to afford evidence of the existence of a localised centromere in their chromosomes are certain algæ (Godward, 1954; King unpublished; Leedale, 1958) and of these a genus fully investigated in regard to its mitotic chromosomes is Spirogyra (Godward, 1950, I953 and unpublished). Spirogyra crassa is a species with twelve relatively large chromosomes. When these are seen in side view at metaphase the chromatids are separate from end to end; they lie parallel to the equator of the spindle and retain this position in anaphase. No distinctive behaviour in regard to the anaphase movements is shown by any region of the chromatid. Although the occurrence of anaphase movement might in itself be regarded as evidence of a centromere function, the movement of other bodies in the spindle at anaphase which has been described for another species of Spirogyra (Godward, 1953) shows that a mechanism of movement which is unrelated to centromeres is present in this species. The movement of the chromosomes is not therefore necessarily brought about as a result of the action of centromeres. Possible interpretations include also a polycentric chromosome for Spirogyra (Godward, 1954) and a "diffuse" centromere for other organisms (Malheiros, de Castro and Camara, 1947; Hughes-Schrader, 1948).

Apart from the role usually ascribed to the centromere in mitosis, an important part is played by it in meiosis in connection with terminalisation of chiasmata (Darlington, 1937, p. 106, " different degrees of terminalisation are . . . due to different degrees of centromere repulsion, in relation to the length of the chromosome".

Meiosis has been seen previously only in species of Spirogyra with very small chromosomes (Tröndle, I9II; Karsten, I908). Observation of meiosis in Spirogyra crassa which has chromosomes up to $7 \mu$ long at metaphase should be valuable in elucidating chiasma behaviour in chromosomes which do not have a localised centromere.

\section{MATERIALS AND METHODS}

Spirogyra crassa appears to be restricted to southern England, and even there it is spasmodic in its appearance in the localities from which it has been collected. It has not been possible to maintain it indefinitely in culture in a reasonably normal condition. Zygospores were available only on three occasions. On the first and second of these it was assumed that meiosis could be found only after a lapse of months or at least weeks after the fusion of gametes. When, however, on the second occasion zygospores only three weeks old were found to contain one large and three 
aborted nuclei it was realised that meiosis must occur earlier. Accordingly in October and November 1956 zygospores were examined a week after fusion of gametes. This was also too late, and when the conjugation was almost over for all the material, zygospores were tested from the time of fusion. It was found that under laboratory conditions, meiosis was begun immediately after fusion of nuclei, which usually took place a day after fusion of gametes. The first metaphase or diakinesis coincides approximately with the process of maturation of the zygospore wall; when the wall is fully matured meiosis is over. Merely finding the time when meiosis takes place took ten years.

Spirogyra crassa (Kützing) Czurda was identified by reference to the characters of the zygospore wall primarily, in conjunction with other usual morphological criteria. The species could also easily have been recognised by its striking cytological characters as they had been described, though incompletely, by previous investigators (e.g. Geitler, I930 ; Van Wisselingh, Igoo).

The iron acetocarmine squash method was employed. Although difficult with such material it was easier than other methods tried.

\section{OBSERVATIONS}

Early prophases although recognised were uninterpretable; it was seen, however, that one enormous nucleolus was always present; in the diploid nucleus this would be associated with the four nucleolarorganising chromosomes.

\section{(i) Diakinesis}

Fairly late diakinesis (pl. I, fig. 5 and text-fig. 3) was the earliest well stained stage. In this there was no trace of nucleolus nor distinction of the nucleolar-organising chromosomes, despite their characteristic appearance at mitotic prophase and metaphase (Godward, 1950). Usually the bivalents were ring-shaped or contained two loops; occasionally three loops were found. These are regarded by the author as evidence of the presence of two, three or four chiasmata. By focussing up and down all four chromatids could be found as separate structures and exchange of partners observed in successive chiasmata. Nevertheless the chromatids are thick and have a woolly or sticky appearance which here, as at later stages, appears to result from the presence of an outer coating of structureless material (Godward, I954) drawn out into radiating projections, which has been interpreted as loose or incomplete deposition of material derived directly or indirectly from the nucleolus. John and Lewis (1957) consider it possible that association of bivalents in Periplaneta takes place by means of " matrical stickiness"; they are uncertain whether chiasmata are present or not. In male Drosophila there are no chiasmata between the autosomes (Darlington, 1934). Here, however, the chromosome behaviour and appearance of bivalents are unlike those of Spirogyra crassa, since in the former organism the "four chromatids . . . in the autosome-pairs . . . lie equally parallel at diakinesis" and "are associated apparently at the centromere, but between diakinesis and metaphase suddenly turn away from one another at this point ".

In Spirogyra a preparation showing a bivalent with three loops was lost in the attempt to make it permanent. The photograph 
(pl. I, fig. 5) of a bivalent with two loops, is perhaps not sufficiently convincing evidence for the existence of chiasmata. Owing to the "woolly" appearance of the chromatids distinct pictures of the conventional kind will not be obtained. As metaphase approaches the woolly appearance is diminished and terminalisation occurs, so that the chromatids separate more distinctly from each other; they do not become quite detached however since the sticky material (or matrix) continues to form connecting strands.

\section{(ii) Metaphase}

Metaphase is recognised by the formation of the spindle and the arrangement of the bivalents on the equator (pl. I, fig. 4). The spindle in side view has the shape of a flattened sphere and it is not easily deformed by pressure. It is composed of numerous coarse fibres, or structures which when stained look like fibres. Although the process of formation of the spindle has not been observed at meiosis, it has at mitosis, when it was composed of nuclear and extra-nuclear components, both partly formed before the disappearance of the nuclear membrane (Godward unpublished).

At metaphase, the bivalents are composed of four parallel chromatids, apparent terminalisation being complete (pl. I, fig. 6; pl. II, figs. 7,8 and 9). The bivalent in side view lies with two chromatids above and two below the equator; all are partly connected by strands of sticky material. The connections between the chromatids above and those below the equator are the first to break down when anaphase occurs (pl. II, figs. 7, 8 and 9, text-fig. 2). If this metaphase and anaphase were interpreted as that of Luzula has been (Malheiros, de Castro and Camara, 1947) then homologous chromosomes lie side by side, sister chromatids one above the other on the equator, and sister chromatids are separated at anaphase I. In Luzula, however, the sister chromatids (or rather, the homologous chromosomes) can be identified as such from their position on the equator-particularly when only one subterminal chiasma is present-since the chromosomes are separated between the chiasmata. In Spirogyra crassa on the other hand at least two apparent chiasmata always remain and the chromosomes are associated by "matrical stickiness" along their length at metaphase I. There is thus no clearcut evidence of the position of sister chromatids (but see anaphase I and discussion, p. 59).

\section{(iii) Anaphase I}

The diagram, text-fig. I, represents an anaphase I with the two chromatid plates seen in polar view as happens in a squash preparation. Pl. II, fig. Io shows one of these chromatid plates-in the permanent preparation the other though still recognisable is damaged. This diagram emphasises the difference between this meiotic anaphase and that of most higher plants and animals, also the identical appearance 
of the two plates; but most of all, the ring-shaped appearance of what may be termed the " half-bivalents". These are pairs of chromatids still loosely associated at the ends; and if the original bivalent had been ring-shaped or had contained the potentiality of this shape by consisting of two homologous chromosomes lying parallel to the equator and associated by two chiasmata one at each end, then, if the bivalent had separated into half-bivalents in such a way that homologous chromatids still associated at the ends passed to opposite poles, the appearance of the resulting anaphase plates would be

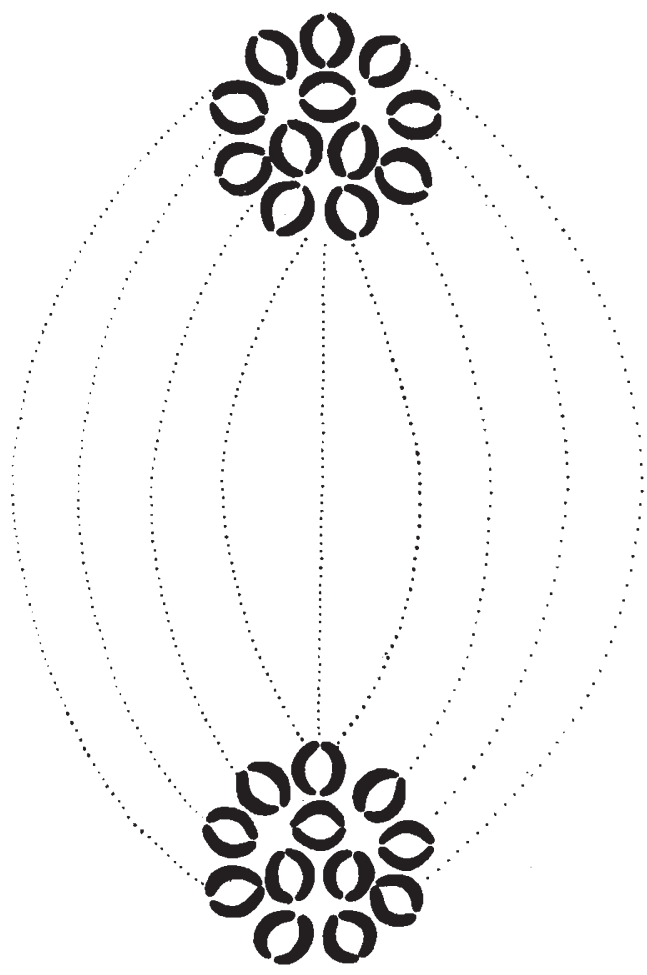

TEXT-FIG. I.-Diagram of anaphase I, showing identical sister anaphase plates of half-bivalents.

exactly what is found here. The position of the homologous chromosomes at metaphase would have been the same as in Luzula. The form of the anaphase $I$ in $S$. crassa is therefore indirect evidence that sister chromatids are separated at this stage and the first meiotic division is equational, as in other organisms without a localised centromere.

At this stage also, as pl. II, fig. Io shows clearly, there are again strands of stainable material stretching from one chromatid to its mate, though not usually reaching it, except that the ends are probably retained in the end-association by this material. Similar strands extend in the same line on the outer side of the chromatids, facing away from the centre of the pair, as if the material forming the outer 
cover of the chromatid were being aligned by forces peculiar to the half-bivalent. Reference to Pl. II, fig. Io will show that the direction of this alignment is different for each (see also text-fig. 2 and discussion, p. 59).

(iv) Metaphase II

Anaphase I passes into metaphase II since there is no interphase. The formation of the spindle of metaphase II has not, however, been clearly observed.

\section{(v) Anaphase II}

Only a preliminary remark can be made about this stage, incompletely observed and requiring confirmation. So far as could be seen under the lower powered lenses of the microscope, before the preparation was damaged, the chromatids had fragmented; whether all or some cannot be definitely stated. It may be recalled here that usually, though not always, three of the resulting nuclei abort.

\section{(vi) The Zygospore}

Most zygospores show for a time one well developed nucleus and three abortive ones, as reported by the original investigators. After three or four weeks only the one nucleus remains. Occasionally a zygospore with two or four fully developed nuclei is found. There was no difficulty about getting spores to germinate; hundreds of germlings were raised. Never more than one germling appeared from a zygospore; and it seems probable that a di- or tetra-nucleate zygospore would give rise to a germling incorporating all the nuclei, which would be separated by septa. This has not been proved to happen. However, irradiated material does develop filaments different portions of which have different karyotypes, without apparent effect on the morphology of the filament as a whole; there seems no reason therefore why it could not occur following meiosis.

Many clones were raised and paired, but the attempt to get conjugation was not successful.

\section{DISCUSSION}

It had previously been shown (Godward, I950, I953, I954) that species of Spirogyra with large chromosomes tended to have even numbers of chromosomes and in certain favourable species (S. triformis, S. sub-echinata) pairs of similar chromosomes could be clearly identified in the mitotic complement. It is now seen that all meiotic association: are bivalents; there are no quadrivalents for example. Therefore it is unlikely that the zygote is anything but a diploid, the filament haploid.

Secondly, what is the evidence regarding the centromeric organisation of the chromosome? That there is no localised centromere is as obvious at meiosis as at mitosis, from the parallel anaphase I separation and from the absence of any place where sister chromatids are 
undivided. "The centromere divides at metaphase in mitosis but not until the second metaphase in meiosis" (Darlington, 1937). By this test there is no evidence for any centromeres, diffuse or multiple, in Spirogyra (pl. II, figs. 8, 9, 10). Previous work had shown (Godward, 1954) that at mitosis there is in prophase a discontinuous staining of the chromosome, giving it at certain stages the appearance of beads on a string. This, it had been thought, in conjunction with the numerous chromocentres visible in resting stage, and bearing in mind the usual relationships between chromocentres and centromeres, might indicate that the chromosomes were polycentric. Fragmentation of

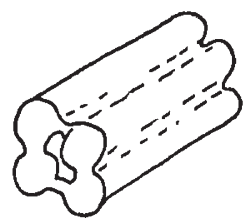

(a)

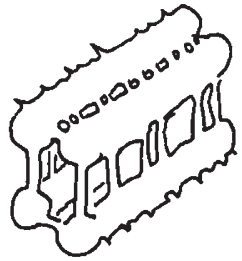

(b)

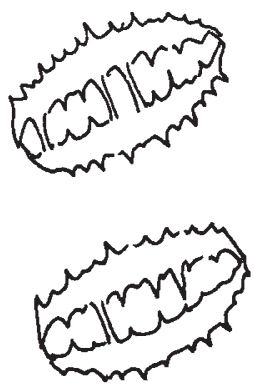

(c)

metaphase 1

anaphase 1

TEXT-FIG. 2.

(a) Diagram showing the bivalent at metaphase. The four chromatids lie side by side adherent by stainable substance. No attempt has been made to show the chiasmata at the ends of the bivalents.

(b) Beginning of anaphase movement, showing the separation of sister chromatids with connecting strands of sticky stainable material; orientation of this material in relation to each separating half-bivalent is also beginning.

(c) Separated half-bivalents at the end of anaphase I. Orientation of stainable substance in relation to each half bivalent is clear, the chromatids are still connected at the ends.

the chromosomes at anaphase II, if confirmed, would tend to uphold this view.

Terminalisation of the two or more chiasmata, if not complete at late diakinesis (pl. I, fig. 5) is always complete at metaphase (pl. II, figs. 8 and 9 and also text-fig. 2). It is not dependent on the repulsion between two localised centromeres in this case. Even if one were to assume that a diffuse centromere or polycentromeres were present, it is difficult to see how terminalisation of two or three chiasmata to the ends of a bivalent could be brought about by repulsion between them, unless that repulsion were to some extent localised. It would seem that Darlington's view (see p. I) of the mechanism by which terminalisation is brought about, is not applicable in this case. Further, the fact that the ends of chromatids still show a residual association after the completion of anaphase (see p. 7)-to put it pictorially, half bivalents show terminalised "half-chiasmata"-indicates that terminalisation has not resulted from pulling forces. 
If the "chiasmata" are really only points of " matrical association" it is still true that there is no evidence of the action of pulling forces associated with a centromere in bringing about changes in this "matrical association",

It is reasonable to assume that here there may be a mechanism responsible both for anaphase movement and in terminalisation of chiasmata, which does not require the assumption of the presence of a centromere.

It is necessary perhaps to refer again to the "woolly" or "sticky" stainable substance, described first by Geitler in Spirogyra (Geitler I 930) and again in some detail by the author (Godward, I953). Critics of the mere existence of such a substance will no doubt regard it as an artifact produced by fixation methods. The amount and peculiar distribution of this substance in Spirogyra precludes any dismissal of it in such terms; if it is an artifact, it is an artifact to be reckoned with. All grades of distribution of the substance are to be observed in the different species, from the mere sticky covering of the chromosome (matrix perhaps) to the actual embedding of the chromosomes in a dense mass of material (Geitler's nucleolar substance), far exceeding the volume of the chromosomes themselves, which accumulates as the nucleolus disappears. Frequently this substance is seen to form part of the spindle; it may proceed in droplets and streamers to the poles, ahead of the chromatids. This latter behaviour clearly demonstrates a mechanism of movement in the spindle, which cannot possibly have any relation to centromeres. An investigation of the substance using radioactive isotopes is being undertaken.

A year or two ago, A. Bajer of Gracow University, Poland, showed an audience at Queen Mary College a film of mitosis taken by polarised light in America, on the microscope built for Shinya Inoué, then at the University of Rochester, N.Y. This showed the centromere emitting a streamer of orientated material at pre-metaphase, the direction of the streamer being independent for each chromosome ( $c f$. the stainable substance in Spirogyra crassa half-bivalents at anaphase I). After orientation on the equator, the chromatids were suddenly pushed apart by the development between them of a mass of birefringent (orientated) material (the phragmoplast). This phenomenon can also be seen although less strikingly on Bajer's more generally known film by phase-contrast illumination. An advancing birefringent zone of no great extent precedes the centromeres, whose function seems perhaps to be the initiation of this birefringent state. The function of the centromere, even the localised centromere of a higher plant, is here seen to be more limited than is generally assumed; it is not necessary to suppose that such a function must be restricted in all cases to a small region of the chromosome. Why, then, in Spirogyra and similarly constituted organisms should one strain after orthodoxy in the search for a centromere?

However, it should be recalled that there is still the possibility 
that Spirogyra and others have a polycentric or compound chromosome-already apparent in Ascaris. If this were proved, then the apparent terminalisation of chiasmata or breaking of some other form of association in the bivalent needs to be looked at in a new way.
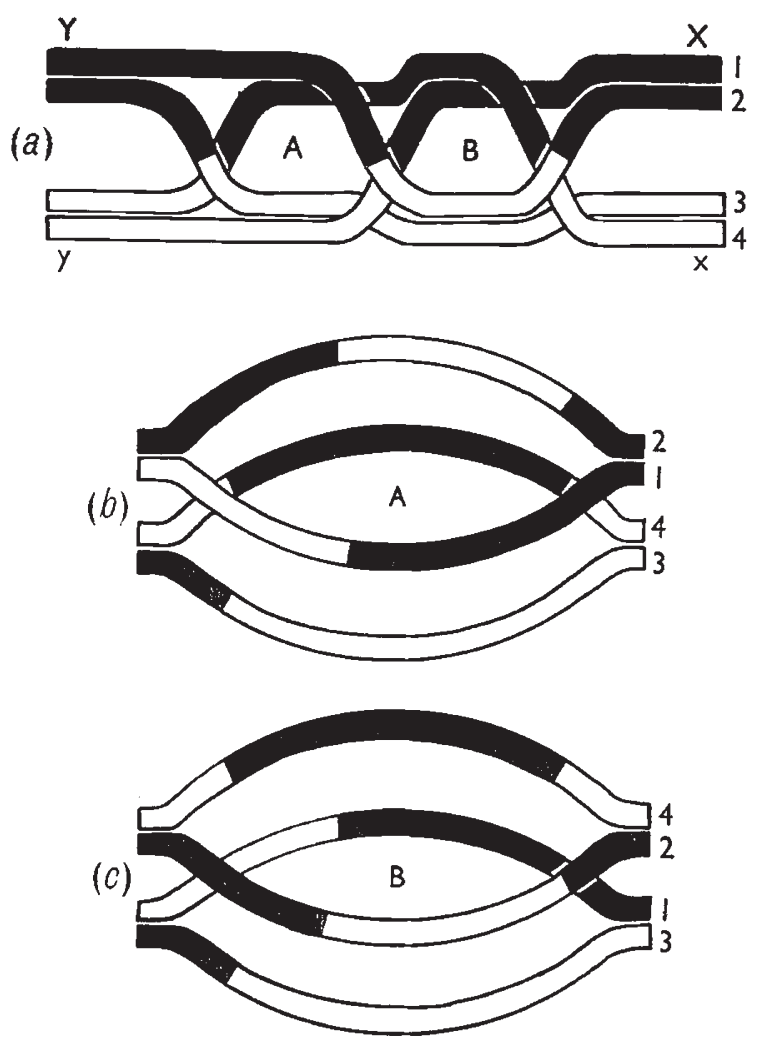

TeXT-FIG. 3 .

(a) Diagram of bivalent at diakinesis, with three chiasmata involving all four chromatids. There are two loops A and B, between the chiasmata. Loci $x$ and $y$ are located as shown; no localised centromere.

(b) Anaphase following opening of loop A with the consequent terminalisation. The division is equational for $y$, reductional for $x$.

(c) Anaphase following opening of loop B. The division is equational for both $x$ and $y$.

Other loci may be imagined in the regions forming loops A and B; whether they segregate at Ist or 2nd anaphase will depend on whether loop A or loop B opens.

Finally, the possibility of testing whether the separation of sister chromatids does occur at the first division of meiosis by genetic means may be discussed briefly. Although the cytological evidence points to such separation, it is by no means conclusive. In Luzula (Malheiros, de Castro and Camara, 1947) there is the occasional rod bivalent, usual in coccids, where the single chiasma is terminalised so that the bivalent lies horizontally on the equator of the spindle, its sister chromatids demonstrably destined to separate at anaphase I. Speaking 
Plate I

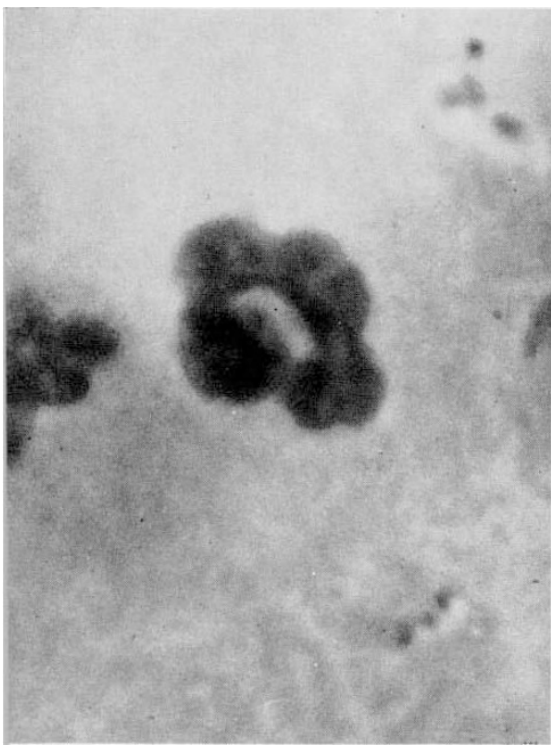

FrG. 6.-Single metaphase bivalent seen in end view. All four chromatids are seen distinctly although strands of stainable material connect them.
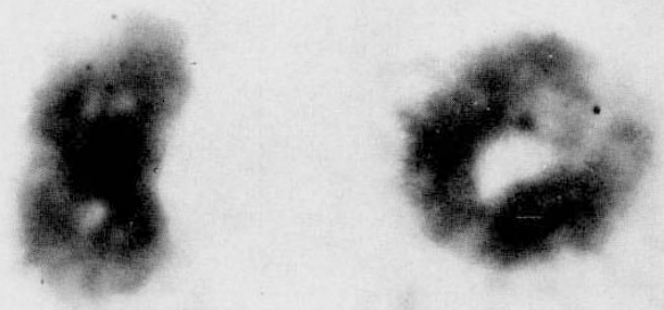

Frc. 5--Late diakinesis. Two bivalents, the left with three chiasmata and two small loops, somewhat obscured by the characteristic diffuse "woolly" outline of the chromosomes, the right with two terminalised chiasmata.

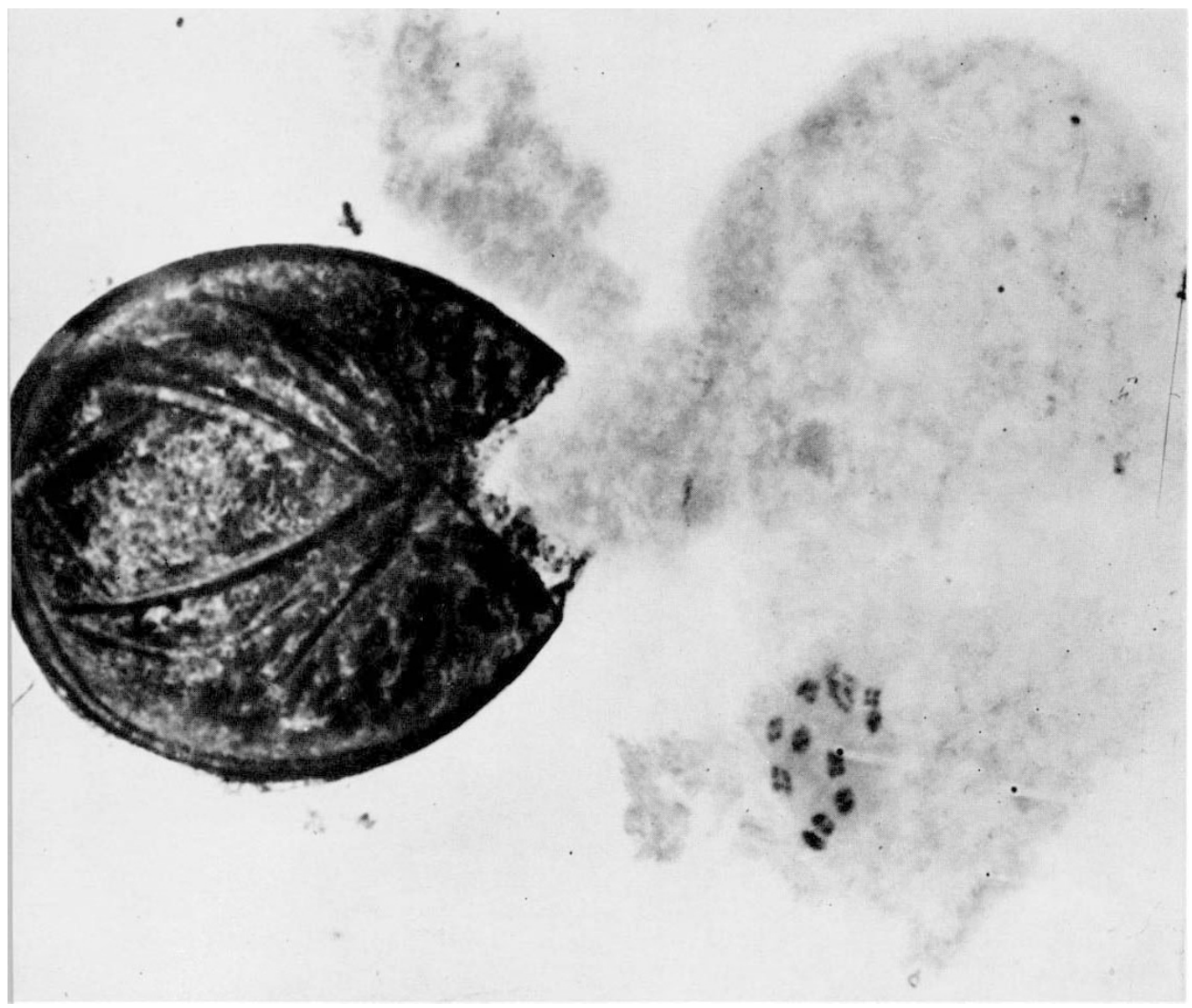

FIG. 4.-Squashed zygospore with metaphase I showing 12 bivalents. 


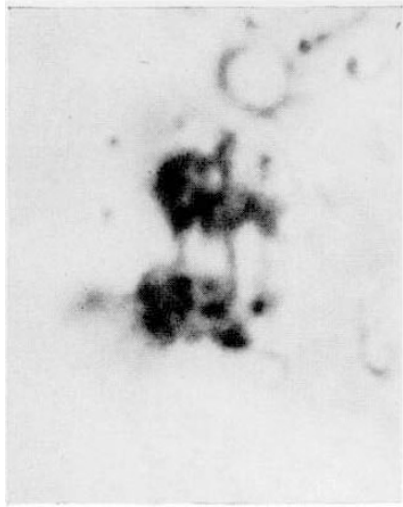

FIG. 9

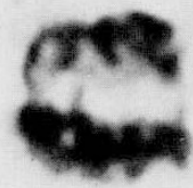

Fig. 8

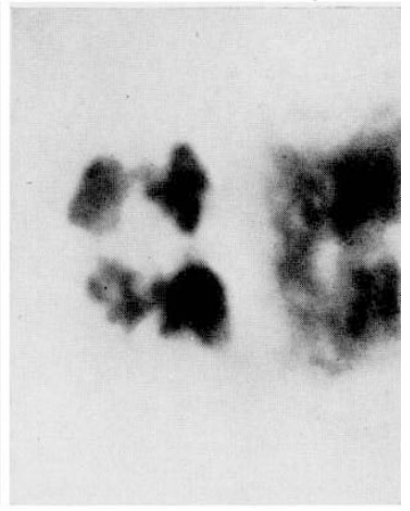

Fig. 7

Figs. 7, 8 and 9.-Bivalents on the equator of the spindle, axis vertical, showing the beginning of anaphase movement. Figs. 8 and 9 side view. Fig. 7 end view of bivalent. Strands of stainable substance are drawn out between (sister) chromatids which lie above one another; non-sisters side by side (see discussion).

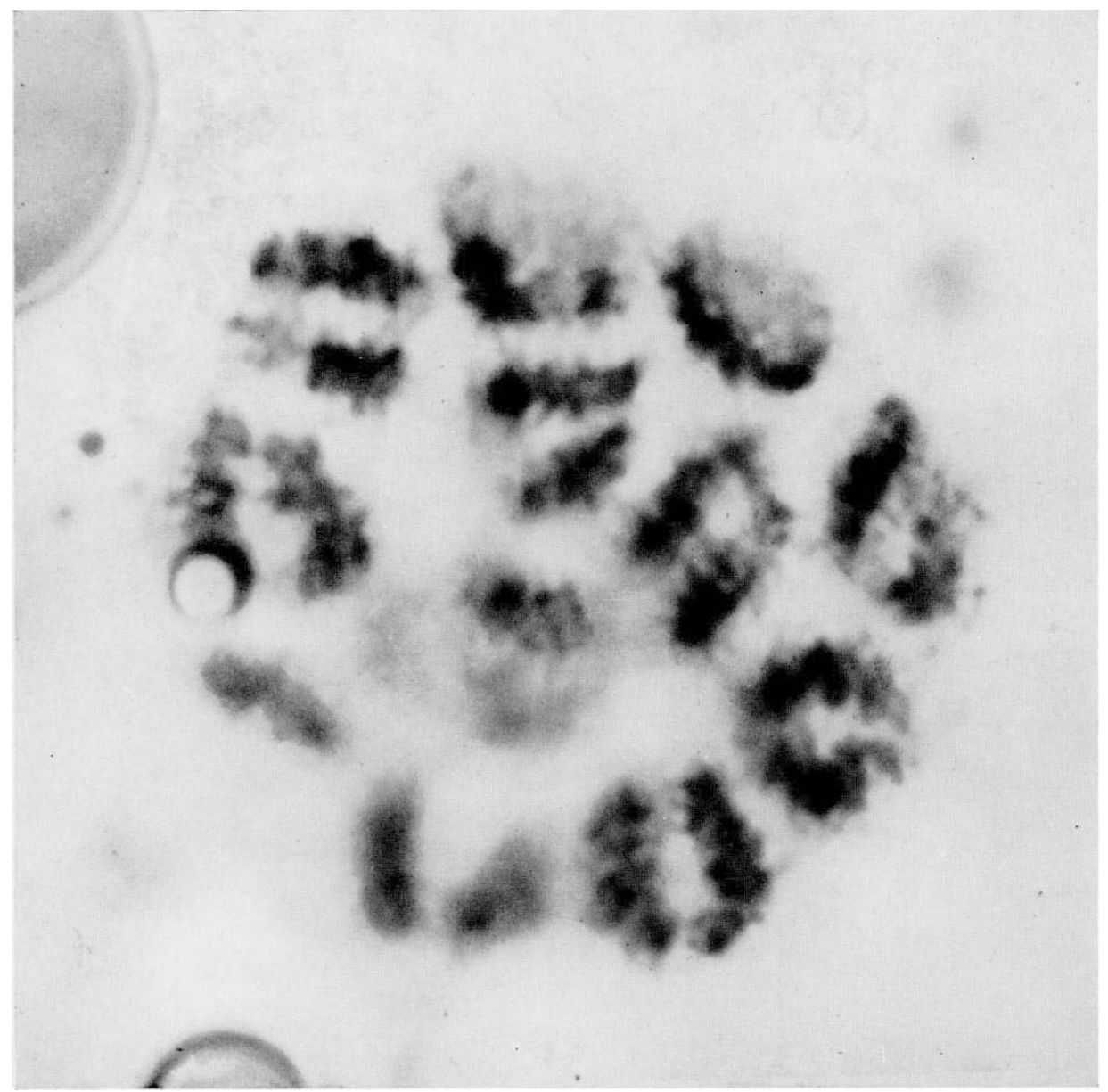

FIG. I0.--Single anaphase plate of chromatids, corresponding to one pole of text-fig. I. Pairs of chromatids form "half-bivalents ; with two exceptions which are out of focus both ends of the chromatids constituting a pair arc still associated. The photograph also shows the diffuse stainable substance on the chromatids orientated in relation to the short axis of each half-bivalent. 
of the coccid-aphid type of meiosis, Hughes-Schrader (1948) says, "The first division . . . is equatorial for all non-crossover regions".

In Spirogyra the situation is more complicated. In text-fig. 3 a simple hypothetical case is shown, where two diplotene loops have been formed between three chiasmata, involving between them all four chromatids. Two loci, $x$ and $y$, have been marked at the ends. In terminalisation either loop A or loop B may open out and be the survivor, while the other is lost as the chiasmata are pushed to the ends. In the absence of a centromere it may be equally likely for loop $\mathrm{A}$ to be the survivor as for loop B. Clearly the segregation of, for example, $x$ or $y$, will be different in the two cases. Where there is a localised centromere, only one segregation of chromatids, in sister pairs, can occur. Where there is none, there are actually three possible segregations of the four chromatids in non-sister pairs. Thus there are more variables affecting the segregation of factors in meiosis where there is no localised centromere and chiasmata are formed.

However, where there are three chiasmata, the number of crossover regions is such, that whatever the centromere situation, the first division is equational for most loci, and the terms sister and nonsister do not mean very much in relation to the chromatid as a whole.

If chiasmata are not formed, there are only the three assortments of the four chromatids in non-sister pairs. It should be possible, by genetic means, to distinguish this situation. It would hardly be possible, however, to find genetic evidence of an equational first division if fig. 3 correctly represents the bivalent.

\section{SUMMARY}

I. Meiosis has been found in Spirogyra, an algal genus whose chromosomes are without localised centromeres, for the first time since I 9 I I and the first critical appraisal of it made.

2. It is shown that terminalisation of chiasmata is not dependent on the presence of a localised centromere. Problems of segregation in the absence of a localised centromere are discussed.

3. It is suggested that centromeric organisation plays no part in the separation of chromatids and that another mechanism must be responsible for this separation in Spirogyra.

Acknowledgments.-The author's thanks are due to Mrs Rosemary Bellamy, her research assistant, for drawing text-figs. I and 3; to Professor D. G. Catcheside and Professor J. M. Thoday for discussion of certain points, and to the Nuffield Foundation for financial support of the research assistant. The microscope used was purchased by the Central Research Fund of the University of London.

\section{REFERENCES}

DARLINGton, C. D. 1934. Anomalous chromosome pairing in the male Drosophila pseudo-obscura. Genetics, 19, 95-1 18.

Darlington, C. D. 1937. Recent Advances in Cytology. Churchill, London. 
GEITLER, L. I930. Uber die Kernteilung von Spirogyra. Arch.f. Protist., 7I, 79-1oo. GODWARD, M. B. E. 1950. Nucleolus and nucleolar-organising chromosomes of Spirogyra. Ann. Bot., N.S. 14, 39-54.

GODWARD, M. B. E. 1953. Geitler's nucleolar substances in Spirogyra. Ann. Bot., N.S. $17,403-416$.

GODWARD, M. B. E. 1954. Diffuse centromere or polycentric chromosomes in Spirogyra. Ann. Bot., N.S. 18 , 143-156.

hUGHES-SCHRAder, s. 1948. Cytology of coccids. Adv. Genet., 2, 127-203.

JOHN, B., AND LEWIS, K. R. 1957. Studies on Periplaneta americana. Heredity, II, I-22. KARSten, G. 1908. Die Entwicklung der Zygoten von Spirogyra jugalis Kutz. Flora, $99, \mathbf{I}-\mathbf{I}$.

xING, G. C. 1960. The cytology of desmids : the chromosomes. Nerw Phyt., 59, 65-72.

LEeDALE, G. F. 1958. Mitosis and chromosome numbers in the Eugleninea. Nature, $181,502-503$.

malHeiros, N., CASTRo, D., AND CAMARA, A. 1947. Cromosomas sem centromero localizado. O casa da Luzula purpurea Link. Agronom. Lusit., 9, $5^{\mathrm{r}-7 \mathrm{I}}$.

TRÖNDLE, A. I9II. Uber die Reduktionsteilung in den Zygoten von Spirogyra. Zeit.f. Bot., 3, 593-6rig.

van WISSELINGH, c. 1900. Uber Kernteilungbei Spirogyra. Flora, 87, 355-377. 